\title{
Serum Levels of Growth Differentiation Factor 11 Are Independently Associated with Low Hemoglobin Values in Hemodialysis Patients
}

\author{
Sho-ichi Yamagishil, ${ }^{1 *}$ Takanori Matsui, Yuka Kurokawa, and Kei Fukami ${ }^{2}$
}

\begin{abstract}
Circulating levels of growth differentiation factor 11 (GDF11) have been shown to decrease with age in several mammalian species, and supplementation of GDF11 by heterochronic parabiosis or systemic administration reverses age-related organ damage. However, there is some controversy about the pathophysiological role of GDF11 in aging-associated organ damage. Since aging process is accelerated in uremia, we compared serum levels of GDF11 in hemodialysis (HD) patients with those in age-matched healthy controls, and then determined the independent clinical correlates of GDF11 in HD subjects. Sixty-two maintenance HD patients (34 male and 28 female; mean age, 52.6 years; mean duration of HD, 7.7 months) were enrolled in the present study. Twenty-nine age-matched subjects were used as a control. GDF11 was measured by a commercially available enzyme-linked immunosorbent assay kit. Serum GDF11 levels in HD patients were significantly higher than those in controls $(9.4 \pm 5.1 \mathrm{pg} / \mathrm{mL}$ vs. $7.3 \pm 5.9 \mathrm{pg} / \mathrm{mL})$. A statistical significance was demonstrated between GDF11 and hemoglobin (inversely). Multiple stepwise regression analysis revealed that hemoglobin $(p<0.001)$ was a sole independent correlate of GDF11 levels in HD patients $\left(R^{2}=0.168\right)$. Our present study suggests that kinetics and regulation of circulating GDF11 may differ between normal physiological aging process and accelerated pathological aging conditions, such as uremia. Given that GDF11 has been shown to inhibit erythroid maturation in mice, elevation of GDF11 levels may be involved in erythropoietin-resistant anemia in HD patients.
\end{abstract}

Key words: aging; cardiology; regeneration

\section{Introduction}

Growth differentiation factor 11 (GDF11), one of the members of bone morphogenetic protein/transforming growth factor- $\beta$ superfamily, is first identified as a regulator of axial skeletal patterning during early embryogenesis, and then found to play a crucial role in various cellular processes, including kidney organogenesis and tissue homeostasis. ${ }^{1-3}$ Recently, systemic levels of GDF11 have been shown to decrease with age in several mammalian species, and supplementation of GDF11 reverses agerelated cardiac hypertrophy, structural and functional derangements of skeletal muscle, and dysfunction of neurogenesis. $^{4-7}$ Furthermore, higher levels of GDF11 and/or its homologue GDF8 are associated with lower risk of cardiovascular events and total mortality in patients with stable ischemic heart disease (IHD). ${ }^{8}$ These observations suggest that GDF11 may be a circulating rejuvenation factor that restores youthful characteristics in various aged organs, thereby being a novel therapeutic target for antiaging medicine. However, there is some controversy about the pathophysiological role of GDF11 in aging-associated organ damage. ${ }^{9-11}$ Indeed, Smith et al. reported that GDF11 did not rescue cardiac hypertrophy in aged mice. ${ }^{9}$ Moreover, GDF11 has been

\footnotetext{
${ }^{1}$ Department of Pathophysiology and Therapeutics of Diabetic Vascular Complications, Kurume University School of Medicine, Kurume, Japan.

${ }^{2}$ Division of Nephrology, Department of Medicine, Kurume University School of Medicine, Kurume, Japan.

*Address correspondence to: Sho-ichi Yamagishi, MD, PhD, Department of Pathophysiology and Therapeutics of Diabetic Vascular Complications, Kurume University School of Medicine, 67 Asahi-machi, Kurume 830-0011, Japan, E-mail: shoichi@med.kurume-u.ac.jp
}

(c) Sho-ichi Yamagishi et al. 2016; Published by Mary Ann Liebert, Inc. This Open Access article is distributed under the terms of the Creative Commons License (http://creativecommons.org/licenses/by/4.0), which permits unrestricted use, distribution, and reproduction in any medium, provided the original work is properly credited. 
shown to increase rather than decrease with age in both rats and humans, and to inhibit toxin-damaged muscle regeneration in old mice. ${ }^{10,11}$

Aging process is accelerated in uremia, and agingrelated organ derangements, including sarcopenia, cardiac hypertrophy, and impaired cognitive function, are more prevalent in uremic patients. ${ }^{12,13}$ Therefore, to clarify the pathophysiological role of GDF11 in humans, it is interesting to examine whether circulating levels of GDF11 are decreased or increased in uremic subjects. In this study, we compared serum levels of GDF11 in hemodialysis (HD) patients with those in age-matched healthy controls, and then determined the independent clinical correlates of GDF11 in subjects on HD.

\section{Subjects and Methods}

Sixty-two maintenance HD patients (34 male and 28 female; mean age, 52.6 years; mean duration of $\mathrm{HD}$, 7.7 months) were enrolled in this study. Patients were dialyzed for $4-5 \mathrm{~h}$ with high-flux dialyzers three times a week. Twenty-nine age-matched subjects were used as a control. Blood pressure was measured in the sitting position using an upright standard sphygmomanometer. Blood was drawn from arteriovenous shunt just before starting HD or from the antecubital veins of controls for determination of blood chemistry as described previously. ${ }^{14}$ GDF11 was measured by a commercially available enzyme-linked immunosorbent assay (ELISA) system (Wuhan EIAab Science Co. Ltd.). Informed consent was obtained from all subjects, and the study protocol was approved by the Institutional Ethics Committees of Kurume University School of Medicine, Japan. This work was conducted in accordance with the Declaration of Helsinki.

Data were presented as mean values \pm standard deviation or medians with the interquartile range. The medication for hypertension and dyslipidemia (renin-angiotensin system [RAS] inhibitors and statins) and the presence or absence of diabetes mellitus were coded as dummy variables. Statistical differences in clinical parameters were determined using Student's $t$-test or chi-squared test. Correlations between GDF11 and other clinical variables were determined by a linear regression analysis. To determine the independent determinants of GDF11, multiple stepwise linear regression analysis was performed. Statistical significance was defined as $p<0.05$. All statistical analyses were performed with the use of the SPSS system (SPSS, Inc.).
Table 1. Clinical Characteristics of Subjects

\begin{tabular}{|c|c|c|c|}
\hline & Controls & HD patients & $p$ \\
\hline No. of patients & 29 & 62 & \\
\hline No. of men/women & $4 / 25$ & $34 / 28$ & $<0.001$ \\
\hline Age (years old) & $56.2 \pm 3.9$ & $52.6 \pm 13.7$ & 0.062 \\
\hline $\begin{array}{l}\text { HD duration (months) } \\
\text { (range) }\end{array}$ & & $7.7 \pm 5.2(0.5-23)$ & \\
\hline Body mass index $\left(\mathrm{kg} / \mathrm{m}^{2}\right)$ & $21.8 \pm 2.4$ & $21.5 \pm 4.7$ & 0.732 \\
\hline Systolic BP (mmHg) & $131.6 \pm 28.50$ & $140.4 \pm 22.0$ & 0.114 \\
\hline Diastolic BP (mmHg) & $75.3 \pm 20.2$ & $82.6 \pm 12.6$ & 0.148 \\
\hline Hemoglobin (g/dL) & $13.6 \pm 2.0$ & $11.2 \pm 1.3$ & $<0.001$ \\
\hline Albumin (g/dL) & $4.5 \pm 0.2$ & $3.6 \pm 0.5$ & $<0.001$ \\
\hline LDL cholesterol (mg/dL) & $120.6 \pm 32.2$ & $87.2 \pm 30.9$ & $<0.001$ \\
\hline Triglycerides (mg/dL) & $82.3 \pm 72.5$ & $128.69 \pm 95.6$ & 0.024 \\
\hline BUN (mg/dL) & $14.0 \pm 3.2$ & $59.5 \pm 12.0$ & $<0.001$ \\
\hline Serum $\mathrm{Cr}(\mathrm{mg} / \mathrm{dL})$ & $0.6 \pm 0.1$ & $11.4 \pm 2.2$ & $<0.001$ \\
\hline Uric acid (mg/dL) & $4.5 \pm 1.1$ & $7.4 \pm 1.0$ & $<0.001$ \\
\hline Corrected Ca (mg/dL) & $8.8 \pm 0.2$ & $9.6 \pm 0.9$ & $<0.001$ \\
\hline Phosphate (mg/dL) & $3.6 \pm 0.5$ & $5.4 \pm 1.1$ & $<0.001$ \\
\hline Intact PTH (pg/mL) & $50.2 \pm 12.9$ & $155.2 \pm 97.0$ & $<0.001$ \\
\hline No. of DM $( \pm)$ & $29 / 0$ & $52 / 10$ & 0.024 \\
\hline HbA1c (\%) & $5.2 \pm 0.5$ & $5.1 \pm 0.8$ & 0.197 \\
\hline hsCRP (mg/dL) & $544 \pm 785$ & $3704 \pm 11681$ & 0.040 \\
\hline GDF11 (pg/mL) & $7.3 \pm 5.9$ & $9.4 \pm 5.1$ & 0.043 \\
\hline \multicolumn{4}{|l|}{ Medication } \\
\hline RAS inhibitors $( \pm)$ & $29 / 0$ & $15 / 47$ & $<0.001$ \\
\hline Statins $( \pm)$ & $29 / 0$ & $60 / 2$ & 0.328 \\
\hline
\end{tabular}

Values are shown as mean \pm standard deviation or range.

$\mathrm{BP}$, blood pressure; BUN, blood urea nitrogen; $\mathrm{Ca}$, calcium; $\mathrm{Cr}$, creatinine; DM, diabetes mellitus; GDF11, growth differentiation factor 11; $\mathrm{HbA1C}$, glycated hemoglobin; HD, hemodialysis; hsCRP, high-sensitivity $C$ reactive protein; $L D L$, low-density lipoprotein; $P T H$, parathyroid hormone; RAS, renin-angiotensin system.

\section{Results}

Table 1 shows the clinical variables of subjects. Serum GDF11 levels in HD patients were significantly higher than those in controls $(9.4 \pm 5.1 \mathrm{pg} / \mathrm{mL}$ vs. $7.3 \pm 5.9 \mathrm{pg} /$ $\mathrm{mL})$. Hemoglobin, albumin, and low-density lipoprotein cholesterol levels were significantly lower in HD patients, whereas triglycerides, blood urea nitrogen creatinine, uric acid, corrected calcium, phosphate, intact parathyroid hormone, and high-sensitivity C-reactive protein values were higher than in control subjects. The number of HD patients with diabetes or who received RAS inhibitors or statins were 10,47 , and 2 , respectively. A statistical significance was demonstrated between GDF11 and hemoglobin (inversely) (Table 2). Multiple stepwise regression analysis revealed that hemoglobin $(p<0.001)$ was a sole independent correlate of GDF11 levels in HD patients $\left(R^{2}=0.168\right)$ (Table 2).

\section{Discussion}

Cardiac hypertrophy and remodeling as well as impaired regeneration of skeletal muscle and neurons in old mice have been reversed by heterochronic parabiosis. ${ }^{4-7}$ Furthermore, circulating levels of GDF11 are 
Table 2. Univariate and Multiple Stepwise Regression Analysis for the Correlates of GDF11 Levels in HD Patients

\begin{tabular}{|c|c|c|c|c|c|c|}
\hline \multirow[b]{2}{*}{ Variables } & \multicolumn{3}{|c|}{ Univariate } & \multicolumn{3}{|c|}{$\begin{array}{l}\text { Multiple stepwise } \\
\text { regression }\end{array}$} \\
\hline & $\beta$ & SE & $p$ & $\beta$ & SE & $p$ \\
\hline Age & -0.177 & 0.047 & 0.168 & & & \\
\hline Sex & -0.043 & 1.315 & 0.738 & & & \\
\hline HD duration & -0.151 & 0.129 & 0.249 & & & \\
\hline Systolic BP & -0.104 & 0.030 & 0.422 & & & \\
\hline Body mass index & -0.131 & 0.140 & 0.312 & & & \\
\hline Hemoglobin & -0.447 & 0.457 & 0.001 & -0.447 & 0.457 & 0.001 \\
\hline Albumin & -0.041 & 1.451 & 0.754 & & & \\
\hline Triglycerides & -0.138 & 0.007 & 0.284 & & & \\
\hline LDL cholesterol & -0.248 & 0.021 & 0.054 & & & \\
\hline BUN & 0.033 & 0.055 & 0.800 & & & \\
\hline Serum $\mathrm{Cr}$ & -0.012 & 0.340 & 0.926 & & & \\
\hline Uric acid & -0.106 & 0.676 & 0.412 & & & \\
\hline Corrected Ca & -0.090 & 0.741 & 0.487 & & & \\
\hline Phosphate & -0.037 & 0.607 & 0.778 & & & \\
\hline Intact PTH & 0.052 & 0.007 & 0.691 & & & \\
\hline $\mathrm{HbA} 1 \mathrm{c}$ & -0.091 & 0.801 & 0.491 & & & \\
\hline DM & -0.115 & 1.770 & 0.372 & & & \\
\hline hsCRP & 0.112 & 0.000 & 0.396 & & & \\
\hline RAS inhibitors & -0.202 & 1.246 & 0.407 & & & \\
\hline Statins & 0.580 & 1.550 & 0.662 & & & \\
\hline
\end{tabular}

$\beta$, standardized regression coefficients. $R^{2}=0.168$.

Male $=0$, female $=1$. Medication $(+)=0$, medication $(-)=1$. $\mathrm{SE}$, standard error.

decreased with age in several animal species, and supplementation of GDF11 has been shown to rescue these age-related organ damage. ${ }^{4-7}$ These observations suggest that GDF11 may be a circulating rejuvenation factor in young mice that could reverse age-related dysfunction of multiple organ systems. ${ }^{4-7}$ However, other groups have reported that GDF11 is increased rather than decreased with age and that reduced circulating levels of GDF11 are unlikely a rejuvenation factor that reverses agedependent changes in mouse heart, skeletal muscle, and brain. ${ }^{9-11}$ Therefore, to further examine the pathophysiological role of GDF11 in human aging process, we chose uremic patients and measured their GDF11 levels in this study because age-related organ damage is accelerated under uremic conditions. ${ }^{12,13}$

We demonstrated here for the first time that circulating GDF11 levels were significantly increased in HD patients than in age-matched controls and that age was not associated with GDF11 levels in uremic subjects. Recently, Fadini et al. reported that compared with control subjects, diabetic patients had significantly increased plasma GDF11 values, especially those with macroangiopathy. ${ }^{15}$ Moreover, they showed that there was no correlation between age and GDF11 in type 2 diabetic subjects. ${ }^{15}$ These findings were in striking contrast with the observation in the Heart and Soul and HUNT3 cohorts, showing that GDF11/8 values were lower in older participants and inversely associated with increased risk of cardiovascular events and overall morality in patients with IHD. ${ }^{8}$ As the case with uremia, given that diabetes is characterized with accelerated aging and increased risk for cardiovascular disease, ${ }^{16,17}$ kinetics and regulation of circulating GDF11 may differ between normal physiological aging process and accelerated pathological aging conditions, such as uremia and diabetes. Elevation of GDF11 levels in uremic patients could be attributed to impaired clearance by $\mathrm{HD}$, which may work as a counter-system against accelerated aging-related organ damage in patients with $\mathrm{HD}$. Another possibility is that difference of reagents and/ or methods used for assaying GDF11 could account for the discrepant results (modified aptamer-based proteomic platform in the Heart and Soul and HUNT3 cohorts vs. ELISA in Fadini's and our subjects).

In this study, we also found that low hemoglobin values were independently associated with high GDF11 levels in HD patients. GDF11 has been shown to inhibit erythroid maturation in mice both in vivo and ex vivo. ${ }^{18,19}$ So increase of GDF11 levels may be involved in erythropoietinresistant anemia, which is often observed in uremic patients. ${ }^{20}$ Since a poor initial hematopoietic response to erythropoietin is associated with adverse cardiovascular events in diabetic patients with uremia, ${ }^{20}$ pharmacological upregulation and/or supplementation of GDF11 may not exert beneficial effects on aging-related disorders, such as cardiovascular disease in HD patients.

\section{Limitations}

There are some limitations in this study. This study was cross-sectional and thus could not assess the questions of whether elevation of GDF11 was a cause or consequence of uremia or anemia. The small sample size is limited for subgroups analysis with solid results. Hemoglobin levels in HD patients are influenced by therapies, such as administration of erythropoiesisstimulating agents and iron. Therefore, various comedications may limit and confound the present findings. Thus, a further study will be needed to address whether supplementation of GDF11 could be functionally correlated with uremia-related organ dysfunction.

\section{Acknowledgment}

This work was supported, in part, by Grants-in-Aid for Scientific Research B (grant number 22390111) (S.Y.) from the Ministry of Education, Culture, Sports, Science, and Technology of Japan. 


\section{Author Disclosure Statement}

No competing financial interests exist.

\section{References}

1. McPherron AC, Lawler AM, Lee SJ. Regulation of anterior/posterior patterning of the axial skeleton by growth/differentiation factor 11. Nat Genet. 1999;22:260-264.

2. Esquela $A F$, Lee SJ. Regulation of metanephric kidney development by growth/differentiation factor 11. Dev Biol. 2003;257:356-370.

3. McPherron AC, Huynh TV, Lee SJ. Redundancy of myostatin and growth/ differentiation factor 11 function. BMC Dev Biol. 2009;9:24.

4. Loffredo FS, Steinhauser ML, Jay SM, et al. Growth differentiation factor 11 is a circulating factor that reverses age-related cardiac hypertrophy. Cell. 2013;153:828-839.

5. Sinha M, Jang YC, Oh J, et al. Restoring systemic GDF11 levels reverses age-related dysfunction in mouse skeletal muscle. Science. 2014;344:649-652.

6. Katsimpardi L, Litterman NK, Schein PA, et al. Vascular and neurogenic rejuvenation of the aging mouse brain by young systemic factors. Science. 2014;344:630-634.

7. Poggioli T, Vujic A, Yang $P$, et al. Circulating growth differentiation factor 11/8 levels decline with age. Circ Res. 2016;118:29-37.

8. Olson KA, Beatty AL, Heidecker B, et al. Association of growth differentiation factor $11 / 8$, putative anti-ageing factor, with cardiovascular outcomes and overall mortality in humans: analysis of the Heart and Soul and HUNT3 cohorts. Eur Heart J. 2015;36:3426-3434.

9. Smith SC, Zhang X, Zhang X, et al. GDF11 does not rescue aging-related pathological hypertrophy. Circ Res. 2015;117:926-932.

10. Egerman MA, Cadena SM, Gilbert JA, et al. GDF11 increases with age and inhibits skeletal muscle regeneration. Cell Metab. 2015;22: 164-174.

11. Rodgers BD, Eldridge JA. Reduced circulating GDF11 is unlikely responsible for age-dependent changes in mouse heart, muscle, and brain. Endocrinology. 2015;156:3885-3888.

12. Kooman JP, Broers NJ, Usvyat L, et al. Out of control: accelerated aging in uremia. Nephrol Dial Transplant. 2013;28:48-54.

13. White WE, Yaqoob MM, Harwood SM. Aging and uremia: is there cellular and molecular crossover? World J Nephrol. 2015;4:19-30.
14. Nagano M, Fukami K, Yamagishi S, et al. Tissue level of advanced glycation end products is an independent determinant of high-sensitivity Creactive protein levels in haemodialysis patients. Nephrology (Carlton). 2011;16:299-303.

15. Fadini GP, Menegazzo L, Bonora BM, et al. Effects of age, diabetes, and vascular disease on growth differentiation factor 11: first-in-human study. Diabetes Care. 2015;38:e118-e119.

16. Yamagishi S, Fukami K, Matsui T. Evaluation of tissue accumulation levels of advanced glycation end products by skin autofluorescence: a novel marker of vascular complications in high-risk patients for cardiovascular disease. Int J Cardiol. 2015;185:263-268.

17. Yamagishi S. Potential clinical utility of advanced glycation end product cross-link breakers in age- and diabetes-associated disorders. Rejuvenation Res. 2012;15:564-572.

18. Dussiot $M$, Maciel TT, Fricot $A$, et al. An activin receptor IIA ligand trap corrects ineffective erythropoiesis in $\beta$-thalassemia. Nat Med. 2014;20:398-407.

19. Suragani RN, Cadena SM, Cawley SM, et al. Transforming growth factor- $\beta$ superfamily ligand trap ACE-536 corrects anemia by promoting late-stage erythropoiesis. Nat Med. 2014;20:408-414.

20. Solomon SD, Uno H, Lewis EF, et al. Erythropoietic response and outcomes in kidney disease and type 2 diabetes. N Engl J Med. 2010;363:1146-1155.

Cite this article as: Yamagishi S, Matsui T, Kurokawa Y, Fukami K (2016) Serum levels of growth differentiation factor 11 are independently associated with low hemoglobin values in hemodialysis patients, BioResearch Open Access 5:1, 155-158, DOI: 10.1089/ biores.2016.0015

$\begin{aligned} & \text { Abbreviations Used } \\ & \text { ELISA }=\text { enzyme-linked immunosorbent assay } \\ & \text { GDF11 = growth differentiation factor } 11 \\ & \mathrm{HD}=\text { hemodialysis } \\ & \mathrm{IHD}=\text { ischemic heart disease } \\ & \mathrm{RAS}=\text { renin-angiotensin system }\end{aligned}$

\section{Publish in BioResearch Open Access}

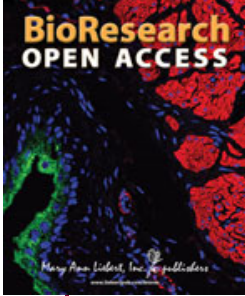

- Broad coverage of biomedical research - Immediate, unrestricted online access - Rigorous peer review - Compliance with open access mandates - Authors retain copyright

- Highly indexed

- Targeted email marketing 